\title{
LV. The resonance spectra of sodium vapour
}

\section{R.W. Wood}

To cite this article: R.W. Wood (1908) LV. The resonance spectra of sodium vapour, Philosophical Magazine Series 6, 15:89, 581-601, DOI: 10.1080/14786440809463801

To link to this article: http://dx.doi.org/10.1080/14786440809463801

册 Published online: 16 Apr 2009.

Submit your article to this journal $\pi$

Џll Article views: 2

Q View related articles ¿

4 Citing articles: 5 View citing articles $\square$ 
THE

\title{
LONDON, EDINBURGH, AND DUBLIN \\ PHILOSOPHICAL MAGAZINE
}

\author{
AND \\ JOURNAL OF SCIENCE.
}

[SIXTH SERIES.]

$M A Y 1908$.

LV. The Resonance Spectra of Sodium Vapour. By R. W. Woon, Professor of Experimental Physies, Johns Hopkins University*.

[Plates XVIII. \& XIX.]

r IHE vapour of sodium, obtained by heating the metallin 1 a highly exhausted steel tube to a temperature of about 400 degrees, yields an absorption spectrum of great complexity. In addition to the $\mathrm{D}$ lines and the other lines (ultraviolet) of the principal series, which come out reversed, we find the entire visible spectrum, with the exception of a very narrow region in the yellow, filled with fine and sharp absorption-lines. This we shall call the channelled absorption spectrum, and we find it divided into two distinct regions, one extending from wave-length 4500 to 5700 , and the other extending from about 5800 to the extreme limit of the red. It is probable that it extends out as far into the infra-red as 10 or $12 \mu$, for recent investigations by the writer, in collaboration with Professor A. Trowbridge of Princeton, have shown that the vapour exhibits heavy absorption in this region, though the bolometer strip was not narrow enough to resolve the lines. This remarkable absorption spectrum was investigated by the writer in collaboration with J.H. Moore a number of years ago ; but the precision which is now being obtained in the work upon the fluorescence of the vapour has made a

* Communicated by the Author.

Phil. Mag. S. 6. Vol. 15. No. 89. May 1908. 
still more accurate stridy of it advisable, and this work has been carried on by Mr. Clinkscales, one of my students, during the past year. Photographs taken with the 21-foot grating in the second-order spectrum, show that it is even more complicated than was originally believed. We find, on the average, from 60 to 70 absorption-lines within a space only 12 Ang. units in width ; in other words, as many as 30 lines in a region no wider than the distance between the $\mathrm{D}$ lines. This means that in the blue-green channelled absorption spectrum, which is about 1200 A.E. in width, there are roughly speaking about 6000 absorption-lines. Taken collectively these lines form themselves into a number of groups, which resemble the groups seen in the absorption spectra of iodine and bromine, and in certain banded emission spectra.

A small portion of the absorption spectrum of sodium vapour in vacuo, taken with the 21-foot grating in the second-order spectrum by Mr. Clinkscales, is reproduced on Pl. XIX. figs. 4 \& 5. The originals have been enlarged about six-fold, and the portions reproduced are thus on a somewhat larger scale than Rowland's large map of the solar spectrum. Each of the strips reproduced is a little less than 20 Angström units in width, or considerably less than the distance between the series lines in the resonance spectra, the spacings of which vary from 36 to 38 units. The strong iron lines in this region appear on the plates for comparison. The absorption-lines are seen to be separated by distances which in some cases are less than $0 \cdot 15$ unit in width. When the resonance spectra have been photographed with the 12-foot grating, an immense amount of information can be obtained by comparing the spectrograms with this magnificent map of the absorption spectrum. This map will enable us to determine whether a given exciting line strikes an absorptionline exactly, or falls midway between two. It must be distinctly understood that the spectrum reproduced is a positive and not a negative of the absorption spectrum. It resembles a bright-line emission spectrum so closely, that I deem it of importance to draw attention to this fact. It is most remarkable that we can absorb portions of a continuous spectrum and leave regions not much over 0.05 or 0.1 of an Angström unit in width, and it would be interesting to examine this residual light, after transmission through the vapour with a Fabry and Perot interferometer. A discussion of this remarkable transmitted spectrum from the point of view of the pulse theory of white light should prove 
interesting, for the light transmitted appears to be as homogeneous as the light in the spectrum of the iron arc.

To attempt to unravel this spectrum, or find any regularities in it by the usual means, is quite out of the question, for the lines are so numerous and so close together that we could pick out series that would conform to any law that we might choose to invent.

As I have shown in a previous paper, however, we possess a very beautiful experimental method of analysing the spectrum, and of determining just which lines belong together; a method, moreover, which may in time yield results which will enable the theoretical physicists to toll us the exact nature of the piece of machinery which we call the sodium molecule.

If we illuminate the vapour with a powerful beam of white light, it becomes strongly fluorescent, emitting a spectrum which I now believe to be the exact counterpart of the absorption spectrum. During the earlier part of the work, before the methods and apparatus had been perfected to the high degree which they have now reached, it was believed that comparatively few of the absorption-lines gave rise to fluorescence, as the fluorescence spectrum could not be photographed with the high dispersion which is now employed. This fluorescent spectrum is obviously of little help to us, for it is quite as complicated as the absorption spectrum. If, however, we throw monochromatic light into the vapour, instead of white light, we observe a very remarkable phenomenon. We now have series of bright lines spaced at very nearly equal intervals along a normal spectrum, and separated by a distance equal to about 37 Angström units. Various series of lines with varying distribution of intensity can be brought out by changing the wave-length of the exciting light. In every case, light of the same wavelength as that of the exciting light is emitted by the vapour, and in addition a large number of other frequencies, which bear a definite relation to each other. In the absence of the exciting light the vapour is non-luminous, that is the electrons, which we may perhaps regard as revolving in concentric rings, do not radiate any energy. The passage of an intense beam of monochromatic radiation through the medium disturbs this non-radiating system in some way, and causes it to emit a series of bright spectrum-lines. The phenomenon can of course be classed under fluorescence if we choose, but as we appear to be dealing with a much more tangible phenomenon than is usually the case, and as these 2 R 2 
spectra are radically different from spectra excited by any other means, I propose that they be named Resonance spectra, since they are without doubt excited by the resonance of one or more of the electrons of the system when monochromatic radiation plays upon it.

During the past year much additional information has been gathered regarding these remarkable spectra, and though the work is by no means completed at the present time, it seems advisable to place on record the results obtained thus far, in view of the fact that theoretical papers (notably those by G. A. Schott) are appearing from time to time on the nature of the radiation emitted by electron systems.

I have already shown that the $D$ lines are present in the emission spectrum when the vapour is excited by light of the same wave-length. This fact is of interest in connexion with statements recently made by some writers that the $D$ lines are never present unless oxidation is going on, and in the air-tight steel tubes, highly exhausted and repeatedly washed out by the hydrogen evolved by the sodium, it is difficult to see how any oxygen can be present.

The very remarkable fact has now been established that the $\mathrm{D}$ lines can be caused to appear in the emission spectrum by stimulating the vapour with a very intense beam of bluegreen light, from which all yellow light has been completely eliminated. This proves that the mechanism which produces the principal series is a part of, or connected in some way with, that very complicated piece of machinery which gives rise to the channelled absorption spectrum with its thousands of lines.

The second point of interest is the discovery of series of equidistant lines in the red fluorescent spectrum, excited by monochromatic red light, the photographic recording of which has been made possible by the panchromatic plates recently placed on the market by Wratten and Wainwright.

The spectrum emitted when the vapour is stimulated by white light has been photographed under more favourable conditions with a large grating, and has been found to possess much more structure than was at first supposed, especially in the yellow-green region, where very little fine detail could be made out in the earlier photographs.

The magnetic-rotation spectrum has been studied with a view of determining whether the rotatory power is positive or negative for the different electrons. The results obtained 
in the red region of the spectrum have been already published *.

The resonance spectra obtained by stimulating the vapour with the radiations from the cadmium and zine quartz arclamps have been photographed with a large grating, and the wave-lengths of the bright lines which form the series have been determined to within a tenth of an Ångström unit, a tremendous step in advance over the work recorded in the earlier paper, in which we could not be sure of the wavelengths to within less than about 2 Ang. units.

Many new sources of monochromatic light have been used, and the prism spectrograph improved by the addition of a new long-focus photographic objective by Cooke. The resonance spectra described in the previous paper have been photographed over again with this improved apparatus, and we now know the wave-lengths of the lines to within a little less than one unit. During the present year they are being photographed over again with a 12-foot concave grating with a collimating lens of six feet focal length. This work, which will take an enormous amount of patience, is being earried on in collaboration with Mr. Felix E. Hackett, Fellow of the Royal University of Dublin.

Familiarity with the preceding paper above referred to will be presupposed in presenting the more recent results, as the details and methods have been already fully described. A few improvements have been made in the apparatus. Owing to the long exposures it has been found necessary to put a heating-coil and thermostat in the large three-prism spectrograph. This keeps the temperature constant to within a tenth of a degree, and improves the definition in no small degree. The telescope-lens has been removed and replaced by a Cooke photographic objective of $3 \frac{1}{2}$ inches aperture and 40 inches focus, which was made to order. This lens was corrected so as to be as nearly as possible achromatic for the spectrum range 4500-5700, within which the resonance spectra, which are being studied, fall. It also gives fair definition over the range of the entire visible spectrum. The wet cotton jackets on the sodium tube have been replaced by tightly wound coils of lead pipe $(5 \mathrm{mms}$. diameter) through which a current of cold water circulates. In working with open air arcs much difficulty was experienced from the wandering of the arc over the surface of the electrodes. The image of the are on this account moved

* "On the existence of Positive Electrons in the Sodium Atom,' Phil. Mag. February 1908. 
about over the aperture in the drum-retort of the sodium tube. The image of the carbons gave some tronble as well, exciting the complete fluorescence spectrum. Both troubles were obviated by focussing the arc on a small round hole in a screen by means of a large double-convex lens, and then throwing an image of the aperture into the sodium retort by means of a second lens. In this way it was possible to keep the white light away from the sodinm vapour, and correct for the shifting of the arc by moving the lamp from side to side. As exposures of seven or eight hours are often necessary, it will be readily seen that the care of the arc is no light labour. A magnetic field was tried to keep the arc. in a fixed position, but nothing appeared to be gained by this expedient.

The spectra excited by the radiations from the cadmium and zinc arcs in exhausted quartz tubes were photographed with a large plane grating in combination with the Cooke lens, and as the wave-lengths of the resonance spectra excited in these two cases have been determined with considerable accuracy, we will discuss them first. In each case the iron-spectrum was photographed in contact with the resonance spectrum.

\section{Cadmiun Excitation.}

The wave-lengths of the lines in the resonance spectra excited by the monochromatic radiations from a cadmiumvapour lamp have been determined to within one or two tenths of an Angström unit. In the previous paper I showed that stimulation of the vapour with the radiation of wavelength 4800 caused it to emit a series of bright lines spaced at very nearly equal intervals along a normal spectrum.

Whether the lines are in reality spaced at equal distances is a matter of considerable importance, since theoretical treatments of the radiation emitted by electron systems disturbed in various ways can only be verified by the test of experiment. Prof. Larmor has pointed out to me in a letter that a non-radiating system of electrons in steady orbital motion, when disturbed by the absorption of a radiation corresponding in frequency to the frequency of one of the electrons, should then emit radiations giving us a series of lines equally spaced along a normal spectrum. We must bear one fact in mind, however. The light emitted by the vapour is obliged to pass through a certain thickness of cooler vapour before reaching the spectroscope, and since the absorption-lines are very closely packed together, it is 
quite possible that the apparent position of an emission-line of finite breadth may be to a slight extent modified by absorption. If, for example, there is a strong absorptionline on the violet side of and close to the emission-line, the eentre of the emission-line will appear a little on the red side of its true position. Very slight departures from the law of equal spacing need not be regarded as fatal. A portion of one of the spectrograms is reproduced on Pl. XIX. fig. 2. Exciting line 5086 shows and 8 or 10 of the resonanoe lines-Comparison spectrum of iron.

The wave-lengths of the lines in the series excited by stimulation at wave-length 4800 are given in the following table, together with the wave-length differences.

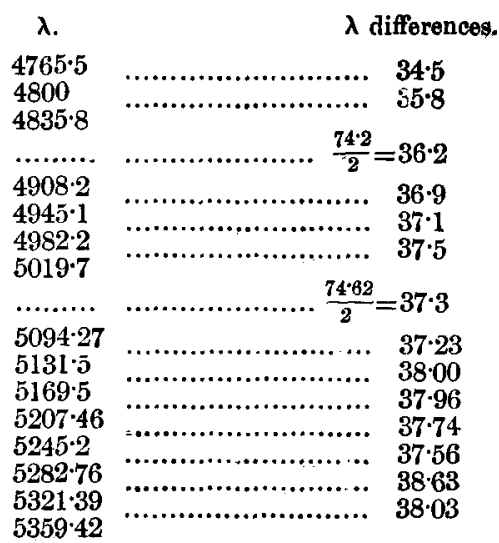

Two only of the lines in the series are missing. In the previous investigation there were three that escaped detection. With longer exposure I feel confident that all wonld appear. It is clear that the lines are closer together in the region of shorter wave-lengths. The slight variations in the differences above the line $5019 \cdot 7$ are, in my opinion, due to the cause mentioned above. If we take the differences between five lines in the series instead of between two, we find the spacing averages remarkably constant. Starting at the top (longest line) of the series, the differences between each line and the fifth below it run as follows : $-151 \cdot 96,151 \cdot 89,151 \cdot 26,150 \cdot 93$, $151 \cdot 46,149 \cdot 8,149 \cdot 3$, and $149 \cdot 17$.

The spectra are now being photographed with the 12-foot grating, and it is quite possible that still greater regularity will result. There seems to be no escape from the fact that the spacing widens slightly as we ascend the spectrum.

We will now consider the resonance spectrum excited by 
the green cadmium-line 5086, which, as I showed in the previous paper, consists of a number of regularly spaced doublets.

The blue lines of the cadmium lamp were cut off by a screen of nitroso-dimethylaniline. In more recent work a screen composed of a concentrated solution of a neodymium salt has been found to work better.

The wave-lengths of the lines excited by the 5086 stimulation are given in the following table :-

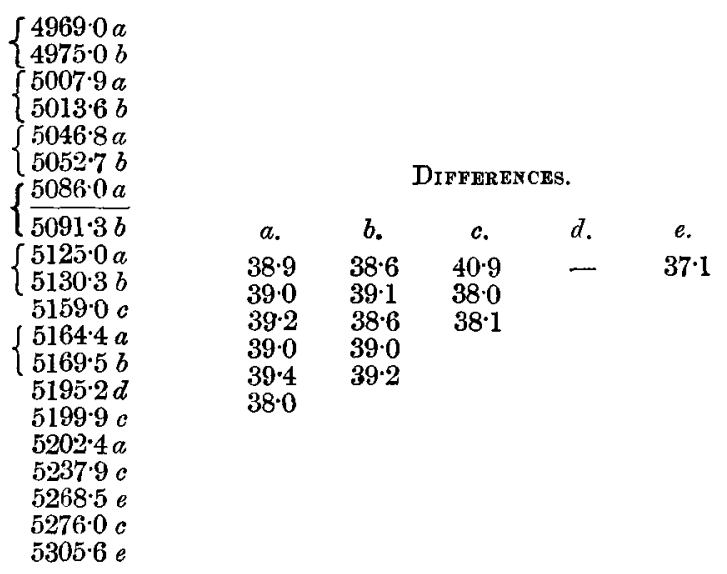

In this table we see that in addition to the doublets which I have spoken of, and which are bracketed, there are a number of other lines. The lines which belong to the same series of equidistant lines are indicated by letters. We find in this case that as we approach the upper limit of the series, other lines appear on the short wave-length side of the line or lines at the upper end, a new series starting where the old one leaves off. This same thing occurs in many other instances, as in the resonance spectrum excited by the barium line 4934 .

It is a little difficult to express in words exactly how these lines come in, and a much better idea can be gained by applying a pair of compasses to the chart and spacing off the series, and noting how the lines of the new series appear on the short wave-length side of the lines which are being spaced off.

I have given the differences between the wave-lengths of the lines of the different series, $a, b, c$, and $e$ in a separate table. The line $d$ appears to be isolated. 


\section{Zinc Excitation.}

The wave-lengths in the resonance spectrum excited by the three blue zinc lines have been determined to within less than 0.2 A.E. Two negatives were made with the large grating and Cooke long-focus lens, one with an exceedingly narrow slit, which was used for the measurements, and one with a wider slit, which is reproduced on Pl. XVIII. fig. 2: the white-light fluorescence above, iron comparison between. On this Plate the lower end of the spectrum did not appear owing to the fact that a corner of a black cloth inside the spectrograph slipped down in front of the end of the plate. The reproduction gives a good idea of the sharpness of the lines in the resonance spectrum, although the exciting lines do not appear on the plate. It must be remembered too that this plate was made with what I have termed a wide slit.

The resonance spectrum contains a large number of series of equidistant lines, though the series are not as extended as in the case of the cadmium excitation and many others which will be considered presently.

I have not, at the present time, photographed the spectra excited by the three lines separately, but by cumparing the plate made with the grating with the one made in the earlier work, I have made a provisional assignment of the lines which I believe belong to the spectrum excited by the longest of the three zinc lines. These lines I have marked with a star in the Table of wave-lengths, and have designated with letters $a, b, c, \& c$., the lines which appear to belong to the same series.

As many lines appear double in the present photographs which appeared as single lines in the earlier spectrograms, it will be understood that too much importance must not be attached to the present assignment of the lines. Many of the series appear to have missing lines, and an attempt is now being made to find them with longer exposures. Exposures of 8 or 10 hours were necessary with the quartz zinc lamp fed by a current of 5 amperes, and the lamps are of very little use after a run of 25 or 30 hours, owing to a black deposit (reduced silica probably) which forms on the inner surface of the tube.

The wave-lengths follow, together with the differences of wave-length of the lines which appear to belong to the same series. The exciting lines are underscored, and the relative intensities are roughly indicated by numerals, 10 meaning very strong, and 1 very faint :- 
Prof. R. W. Wood on the

$\mathbf{5 1 6 8} 8 \quad 1$

$5181 \cdot 11$

$5184 \cdot 2 \quad 1$

$5186 \cdot 8 \quad 6 * b$

$5196 \cdot 01 d$

$5215 \cdot 32 c$

$5220 \cdot 61$

$5225 \cdot 8 \quad 4 * b$

$5232 \cdot 0 \quad 3 * d$

5244.01

$5248 \cdot 7$

$5252 \cdot 81 * c$

$5253 \cdot 72$

$5264 \cdot 5 \quad 2 * b$

$5268 \cdot 0 \quad 1 d$

$5273 \cdot 01$

$5277 \cdot 6 \quad 1$

$5290 \cdot 29 * 0$

$5300 \cdot 0 \quad 1 *$

$5303 \cdot 1 \quad 2 * b, d$

$5305 \cdot 62$

5307.51

$5313 \cdot 7 \quad 2$

$5315 \cdot 8 \quad 1$

$5321 \cdot 1 \quad 3$

$5327 \cdot 0 \quad 3$

$5328.56 * 0$

5338-1 $8 * d$

5350.52

$5351 \cdot 64$

5356.51

$5366.01 c$

$5370 \cdot 01$

$5373.5 \quad 8 * d$

5375.95

$5379 \cdot 2 \quad 1$

$5386 \cdot 12$

$5387 \cdot 4 \quad 5$

$5390 \cdot 2 \quad 2$

$5391 \cdot 9 \quad 3$

$5397 \cdot 3 \quad 1$

$5349 \cdot 1 \quad 2$

$5403 \cdot 3 \quad 4$

$5404 \cdot 3 \quad 2$

$5406 \cdot 3 \quad 1$

$5408.58 d$

Differhinchs.

$b$.

$a$

36.0

$\frac{75 \cdot 4}{2}=37 \cdot 7$

$38 \cdot 2$

$36 \cdot 4$

$\frac{76 \cdot 6}{2}=38 \cdot 3$

$37 \cdot 9$

$\begin{array}{rl}\frac{73 \cdot 4}{2}=36 \cdot 7 & 39 \cdot 0 \\ 35 \cdot 9 & 38 \cdot 7 \\ & 38 \cdot 6\end{array}$

$\begin{array}{rl}\frac{73 \cdot 4}{2}=36 \cdot 7 & 39 \cdot 0 \\ 35 \cdot 9 & 38 \cdot 7 \\ & 38 \cdot 6\end{array}$

$\begin{array}{rl}\frac{73 \cdot 4}{2}=36 \cdot 7 & 39 \cdot 0 \\ 35 \cdot 9 & 38 \cdot 7 \\ & 38 \cdot 6\end{array}$

37.5

$36 \cdot 0$

$37.4 \quad 36.0$

$38 \cdot 3 \quad 35 \cdot 1$

$38 \cdot 5 \quad 35 \cdot 1$

35. 4

$35 \cdot 0$

$34 \cdot 2$

$35 \cdot 3$

$34 \cdot 5$

$5 \cdot 4$
$5410 \cdot 0$ *

5414.03

$5418 \cdot 41$

$5420 \cdot 0 \quad 1$

$5421 \cdot 8 \quad 6$

$5+23 \cdot 3 \quad 6$

$5427 \cdot 6 \quad 2$

$5432 \cdot 2 \quad 2$

$5438 \cdot 06$

$5440 \cdot 31$

$5442 \cdot 73 d$

5443.95

$5448 \cdot 2 \quad 2$

$5451 \cdot 5 \quad 5$

$5454^{\circ} 48$

$5457 \cdot 3 \quad 3$

5458.81

$5465 \cdot 12$

$5471 \cdot 7 \quad 4$

547582

$5478 \cdot 0 \quad 3 d$

$5482 \cdot 64$

$5484 \cdot 1 \quad 5$

5486.97

$5490 \cdot 0 \quad 3$

5494:1 1

$5496 \cdot 2 \quad 3$

5502.02

$5505 \cdot 62$

$5512 \cdot 51 d$

5512.92

$5516 \cdot 1 \quad 3$

5519.3 2

5521.72

$5526 \cdot 7 \quad 1$

$5532 \cdot 61$

$5535 \cdot 22$

5538.0 2

5547.81

$5557 \cdot 6 \quad 4$

5558.56

$5563 \cdot 74$

558312

$5584 \cdot 4 \quad 3$

5588.92

5 
The series are not as pronounced in the case of the zinc excitation as in many of the other cases. Apparently the series which I have marked " $a$ " and which is excited by the longest of the three zinc lines, appears to have its upper members excited by one of the other zinc lines, and not by the line which starts the series. In other words, the line $4883 \cdot 6$ seems to be the last line of the " $a$ " series excited by Zn $4810^{\cdot} 7$, but we find a number of lines in the spectrum excited by the total radiation of the zinc lamp, which form $a$ continuation of this series. Neither of the other zino lines, however, falls at points determined by extending the series down to their region. We must remember, however, that when we stimulate the vapour in the blue region, the series which starts at the point of stimulation gradually dies out as we go up the spectrum, and other series make their appearance, a circumstance which explains the apparent diffioulty just mentioned. There seems to be some evidenoe that as we ascend the spectrum the spacing between the lines of a series begins to grow less again, that is the spacing has a maximum value somewhere in the middle of the spectrum. Compare the series " $a$ " and " $d$ " for example. Too much importance must not be attached to these tables of differences, however, as the lines which belong together have been determined only from the older spectrograms, which are very unsatisfactory, and by spacing off the new spectrum chart with a pair of compasses. The resonance spectrum has now been photographed with the 12-foot concave grating, and as soon as the new zinc lamps arrive the spectra excited by the isolated zinc lines will be recorded with the same instrument.

\section{Lithium Excitation.}

The light of the lithium arc stimulates a most remarkable group of resonance spectra. It is the only means that I have yet found of exciting definite series of lines in the red region of the fluorescence spectrum. There are four exciting lines. in all, but they are so widely separated that the resonance spectra excited by them only overlap to a very slight degree. A photograph taken on a panchromatic plate of the complete spectrum excited by the lithium arc is reproduced on Pl. XVIII. fig. 1. T'he points of excitation are indicated by arrows.

The blue line $(\lambda=4603)$ excites a resonance spectrum consisting of a nearly equidistant group of lines in the violet, and another group in the yellow-green. These two groups are indicated by the brackets "A."

The blue-green line $(\lambda=4972)$ excites a remarkable series 
of lines midway between the groups "A." This series is of especial interest, for, as will appear presently, the barium line 4934 takes hold of the same series, at the first line below the exciting line of the lithium series. The red and orange lines of lithium also excite two well-marked series in the red region of the spectrum, the individual lines of which appear to be very nearly equidistant. As yet I have not determined the wave-lengths of these lines, though they can be determined at any time from the plate which has an iron comparison spectrum running parallel to the resonance spectra. Taken as a whole, this plate is the most beautiful illustration of resonance spectra excited by monochromatic stimulations, that I have obtained up to the present time. The photograph was made with a small two-prism spectrograph, and has not been used for wave-length determinations owing to the small dispersion and the great width of the slit. Spectrograms best suited to the accurate measurement of the lines do not reproduce very well, consequently I have used only those taken with a wide slit for purposes of illustration, and the possible accuracy of the wave-length determinations must not be judged from the photographs which accompany this paper.

We will now consider the series excited by the green-blue line 4972. The wave-lengths of the lines in this series are recorded in the following table. The wave-lengths I consider accurate to about three tenths of an Angström unit, as the plate was an especially good one, taken with a very fine slit.

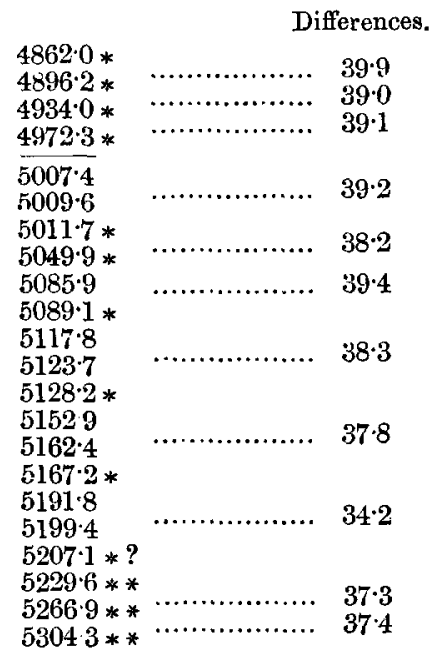


In this table I have marked the lines which form the equidistant set with a $\operatorname{star} *$. The last three belong to a different series not continuous with the first. It will be seen that as we go up the spectrum other lines come in on the short wavelength side of the principal lines, as in the case of the series of doublets excited by the green cadmium line.

A discussion of the spectrum excited by the blue-violet lithium line will be postponed for the present, as I prefer to wait until photographs have been obtained with the large concave grating.

\section{Barium Excitation.}

The excitation by the strong barium line 4934 is of great interest as the same series is brought out as in the case of the lithium excitation. The point of excitation is at the first line below to the exciting line of lithium, and the resonance spectra are identical except that the companion lines which appear on the short wave-length side of the principal lines are not identical. The wave-lengths are given in the following table, the principal lines being marked with stars.

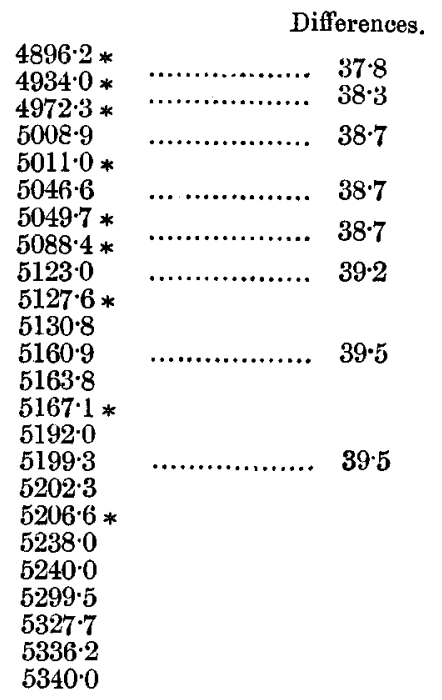

The three strong lines of a different series, which appeared with the lithium excitation (double starred in the table), do. not appear with the barium excitation. 


\section{Thallium Excitation.}

The great line of the thallium arc excites a resonance series of lines some of which are accompanied by companion lines on the short wave-length side. A photograph of the spectrum, taken with a wide slit, is reproduced on PI. XIX. fig. 1. The wave-lengths are as follows :-

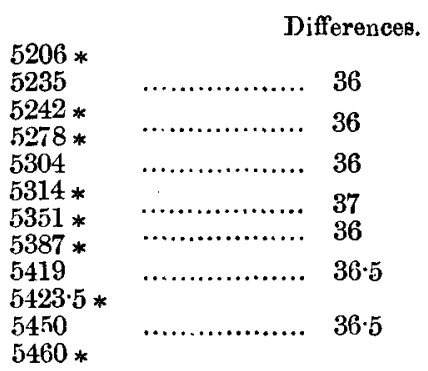

The thallium arc burnt very steadily. The lower carbon, which was bored with a hole $2 \mathrm{mms}$. in diameter, was kept full of metallic thallium, and used as the anode.

The lines of the series are a little closer together in this case, as appears from the table of differences.

\section{Magnesium Excitation.}

A great deal of time was wasted in trying to obtain a satisfactory magnesium are between metallic electrodes in hydrogen at low pressure. It was finally found that the simple expedient of introducing magnesium powder into a hollow carbon answered every purpose, the arc burning steadily. Fresh powder was put in every four or five minutes.

The green triplet ( $b$ group) excite brilliant resonance spectra, which are reproduced on Pl. XVIII. fig. 4 . In this spectrum we find the triplet reproduced more or less perfectly at regular intervals. If the spacing of the individual lines forming the series was the same for each series, it is clear that we should have the triplet reproduced over and over again along the spectrum. That this is so only to an imperfect degree is due to the difference in the spacing of the lines of the different series. No means has as yet been found of exciting the spectra separately, owing to the proximity of the three exciting lines. 
The wave-lengths are recorded below.

It is easy to pick out the three series of equidistant lines excited by the three magnesium lines, but it is impossible to tell anything about the extra lines or to which series any one of them belongs. Lines belonging to the same series are indicated by the same letter, while the exciting lines are underscored. The differences for the three series $a, b$, and $c$ are given in a separate table. The series " $a$ " is the best one obtained, so far as constancy of wave-length difference goes. The slight discrepancy at the middle of the table compensates itself, and is doubtless due to an error of half an Angström unit in the determination of one of the lines.

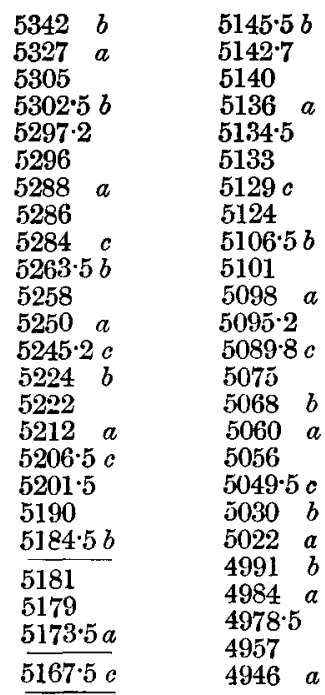

Differences.

\begin{tabular}{lll}
$a$. & \multicolumn{1}{c}{$b}$. & $c$. \\
39 & $39 \cdot 5$ & $38 \cdot 8$ \\
38 & 39 & $38 \cdot 7$ \\
38 & $39 \cdot 5$ & 39 \\
$38 \cdot 5$ & $39 \cdot 5$ & $38 \cdot 5$ \\
$37 \cdot 5$ & 39 & $39 \cdot 2$ \\
38 & 39 & 40 \\
38 & $38 \cdot 5$ & \\
38 & 38 & \\
38 & 39 &
\end{tabular}

\section{Lead Excitation.}

The lead arc causes the sodium vapour to emit a wellmarked series of lines, with a wave-length difference of 39 Ångström units. These lines are accompanied by companion lines sometimes on one side and sometimes on both sides, as will be seen from the chart. The law governing the distribution of the companion lines and the possible cause of their appearance cannot be determined until a larger collection of photographs of the resonance spectra has been made and the results compared and tabulated. 
The lines which form the series are marked with a star.

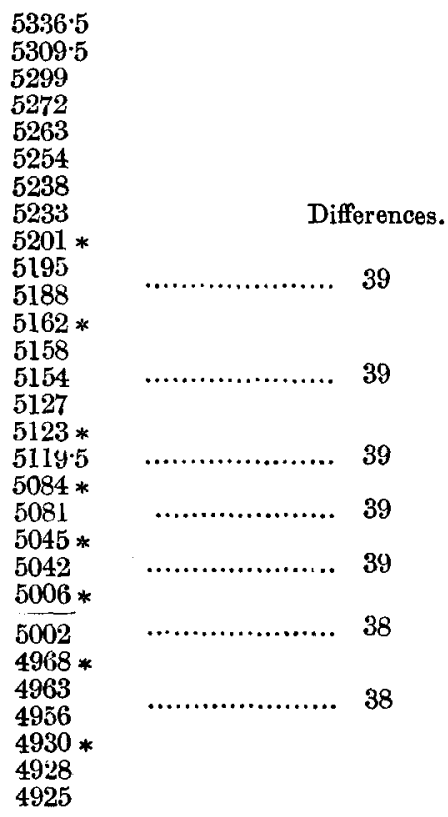

The line in the lead spectrum which stimulates this resonance spectrum has a wave-length of 5006 : there is in addition a weaker line at 5002, and I am not sure whether the light of this line is responsible for any of the spectrum. It comes within one Angström unit of fitting into the series, but it is much fainter than the 5006 line.

Bismuth Excitation.

The bismuth arc is by far the best exciter of the vapour which we have, and it furnishes the most typical resonance spectrum. It has but a single line which is operative, and this line is located in the remote blue. The resonance spectrum consists of a series in the blue, which with long exposures can be traced well up into the green, and a host of lines at the upper end of the spectrum in the yellow-green region.

The exciting line in the resonance spectrum is accompanied by two faint companions, one on each side, the first line above it is a close doublet, the second a wider one, and the first line below is a suspected doublet, judging by its width. In all probability the second and third lines below the exciting 
line are doublets, though they are so faint as to be only just discernible on the photographs obtained thus far.

The wave-lengths in the blue and green region are given in the following table. Two lines in the series appear to be missing.

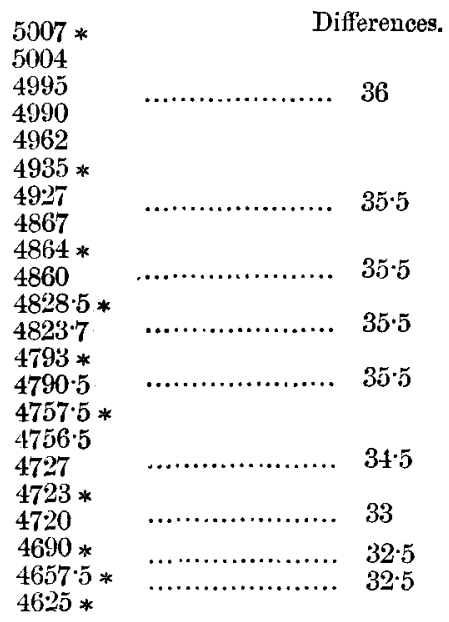

The lines in the yellow-green region are given below. The intensities are designated roughly by numerals, 1 meaning very faint.

$\begin{array}{ll}\mathbf{5 5 3 4 \cdot 4} & 1 \\ \mathbf{5 5 3 0 \cdot 3} & 1 \\ 5509 & \mathbf{1} \\ 5504 & 3 \\ 5500 \cdot 9 & \mathbf{6} \\ 5499 \cdot 5 & 1 \\ 5491 \cdot 6 & \mathbf{2} \\ 5490 \cdot 1 & 2 \\ 5488 \cdot 7 & 5 \\ 5474 \cdot 5 & 5 \\ \mathbf{5 4 7 3} \cdot 5 & 6 \\ 5470 \cdot 5 & 2 \\ 5469 \cdot 0 & 2 \\ 5463 \cdot 8 & 1 \\ 5462 \cdot 3 & 1 \\ 5459 \cdot 4 & 7 \\ \mathbf{5 4 5 8} \cdot 0 & 3 \\ 5456 \cdot 5 & 5\end{array}$

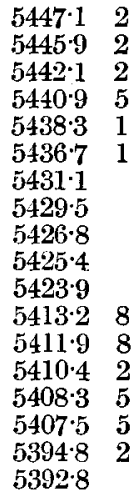

$5391 \cdot 9$

$5391 \cdot 1 \quad 2$

$5388 \cdot 31$

$5387 \cdot 1 \quad 1$

$5379 \cdot 14$

$5377 \cdot 7 \quad 4$

5376.21

$5374.0 \quad 5$

$5373 \cdot 5 \quad 7$

5372551

$5358 \quad 2$

$5357 \cdot 32$

535577

$5336.8 \quad 6$

$5335 \cdot 6$

$5334 \cdot 1 \quad 1$

529982

\section{Silver Excitation.}

In the earlier work it was believed that the silver line 5209 excited the same series as the cadmium line 4800 . This is now known to be an error due to the insufficient Phil. Mag. Ser. 6. Vol. 15. No. 89. May 1908. 2 S 
power of the spectroscope employed. The silver line excites a series, the individual lines of which are about 1.5 Angström units longer than the corresponding lines excited by the cadmium line. The silver arc employed in exciting the resonance spectrum was formed between carbons, the lower one of which was furnished with a core of metallic silver. No prismatic separation was required, as the line 5209 was found to be the only one capable of exciting the vapour. The wave-lengths of the lines in the resonance spectrum are given in the following table. The first six lines in the table were very faint, and their wave-lengths are not so accurate as is the case with the remainder.

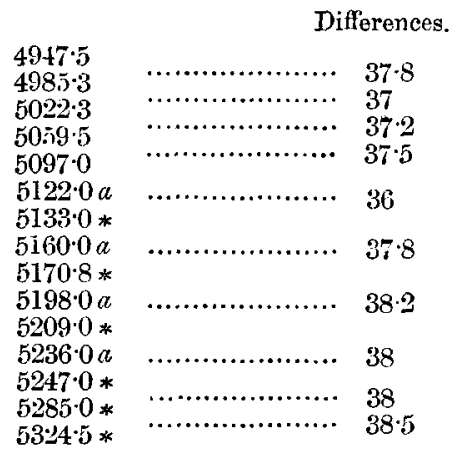

The strong lines which have been measured with considerable accuracy, and which form a series, I have indicated with stars. The first five lines in the table also belong to the same series I think, though there seems to be a slight discrepancy in the table of differences. Four of the strong, equidistant lines are accompanied by faint lines on the short wave-length side. These four companion lines are also equidistant. They are marked " $a$ " in the table, and their wave-length differences are exactly 38 A.E.

\section{Copper Excitation.}

The resonance spectra excited by the light of the arc between electrodes of metallic copper are reproduced on Pl. XVIII. fig. 3. The three green lines are alone operative, and though the line 5293 appears on the plate I am of the opinion that no fluorescence is excited by it. Spectrograms have been obtained of the spectra excited by the three lines separately, but they are not very satisfactory, as the slits of the monochromatic illuminator had to be made very narrow and there was but little light available. To separate close 
lines and still have enough light to work with is now the chief difficulty. I have recently found a chlorate of potash film which in transmitted light shows a very black interference band not much over 15 Angström units in width. The position of this band vacies with the angle of incidence of the light, and it can be set so as to cut off either one of the copper lines at will. It cannot, however, do this for a divergent beam of light, owing to the variation of the incidence angle in this case; and as the crystal is less than a square centimetre in area, little or nothing is gained by its use over the monochromatic illuminator. If we could prepare crystal flakes similar to this, measuring several cms. in width, they could be used with light made parallel by a lens, and the problem of separating spectrum lines would be solved.

In the resonance spectrum excited by the three green copper lines I have already measured over one hundred and twenty-five lines, but until more satisfactory spectrograms have been obtained with the separated radiations I prefer not to publish this table. The spectrum is very complicated, and I do not feel at all sure of the lines which belong together, although there are a number of very well marked series. My surmise of a year ago that the shortest of the three lines excited a series of doublets has been verified.

\section{The Magnetic Rotation Spectmum.}

The present work has shown that there are not many coincidences between the bright lines of the magnetic rotation spectrum and the lines of the resonance spectra. The belief that these coincidences existed, which was expressed in the carlier paper, was due to the low dispersion used. There appear to be six or more series in the magnetic spectrum, but they do not coincide with any of the series excited by monochromatic radiations. The lines which show the magnetic rotation are the lines which come out with especial prominence in the spectrum of the fluorescence excited by white light. At the present time I am engaged in a study of the magnetic spectrum by the method described in my recent paper on the possible existence of positive electrons in the sodium atom.

I have photographed the spectrum over again under more favourable conditions, and have obtained a much larger number of lines, nearly 200 in all, The table of their wavelengths will not be published until the final paper is ready for publication, as I have not yet determined whether all of the lines rotate the plane of polarization in the same direction. 


\section{White Light and Cathode-ray Excitation.}

At the close of the present year an atfempt will be made to photograph the white-light fluorescence spectrum with the 21-foot grating in the second-order spectrum. This will give us a record on the same scale as the absorption-spectrum map. I have already found that the structure is much more complicated than was at first supposed, the darker regions between the strong lines being filled with a multitude of fine lines. The structure of the spectrum which I attempted to show with a drawing in one of the earlier papers is quite incorrect, the peculiar appearance being due to a peculiar distribution of the light in the spectrum, combined with a rather wide slit. The white-light fluorescence spectrum, which begins at about 4600 , ends quite abruptly at the point where the yellow-green doublet of the first subordinate series appears in the arc spectrum of the metal.

This may be an accident of course, but it may also be due to some relation between the parts of the vibrating mechanism, with which we are at present unfamiliar. The general appearance of the upper half of this spectrum is shown on Pl. XVIII. fig. 2 (upper spectrum), with an iron comparison spectrum. The upper end of this spectrum is also shown on Pl. XIX. fig. 3 (upper spectrum), together with the D lines which come out bright, and a portion of the red and orange fluorescence spectrum. The red-orange fluorescence extends a little below the $\mathrm{D}$ lines, but not quite down to the terminus of the yellow-green fluorescence, the region between the two spectra being destitute of light. The bands which border the edge of this black region give it the appearance of a deep chasm. I have already obtained some excellent photographs of this region, and they will be published with the final paper. Exciting the vapour with cathode rays causes it to emit a very remarkable spectrum. The lines of the principal and subordinate series come out with great intensity, as well as the lines which appear in the fluorescence excited by white light. In addition there is an entirely new spectrum, which in a way appears to be symmetrical about the $D$ lines, at least in their immediate vicinity. This spectrum is shown on PI. XIX. fig. 3 (lower spectrum). It is on the same scale as, and placed in coincidence with, the white-light fluorescence spectrum. The lines of the subordinate series $I$ have marked with black dots. It is a very remarkable fact that the bright bands to the right and left of the D lines coincide with broad dark bands in the fluorescence spectrum. This scarcely shows on the print which I am reproducing, and it will in all probability disappear completely in the process of repro- 
duction. I have some plates made a year ago which show the bands most beautifully, but I have not yet enlarged them, and they are on too small a scale to reproduce well.

The present paper must not be regarded as a complete presentation of the subject,and I am publishing these results now in order that they may serve as a guide to those who are at work at the theory of the radiation of electron systems, from the theoretical side.

IVI. The Distritution in Electric Fields of the Active Deposits of Radium, Thorium, and Actinium. By Sidney Russ, Demonstrator of Physics, Victoria University, Manchester*

\section{Introduction.}

THE distribution in an electric field of the excited activity produced by thorium and radium emanations has been studied by several observers. Working with thorium emanation, Rutherford + has shown that the amount of activity imparted to a rod charged negatively decreases as the pressure in the containing vessel is reduced after a certain pressure is reached; while experiments made by Makower $\ddagger$ show that similar effects are obtained with the excited activity produced from radium emanation. Some further experiments by Rutherford § with the emanation from radium indicate that while at atmospheric pressure the greater part of the excited activity is directed to a cathode by moderate electric fields, yet a small fraction (about 5 per cent.) goes to an anode. Reasons are then (loc. cit.) given for supposing that some of the active deposit particles carry a negative charge, thus accounting for their transmission to the anode.

It seemed of interest then to find out whether the quantity of active deposit that goes to an anode changes when the pressure is varied.

A comparison has therefore been made over a range of pressure extending from $001 \mathrm{~cm}$. to $10 \mathrm{cms}$. between the amount of activity imparted to a rod charged positively and then negatively when exposed for the same interval of time to equal quantities of the radium emanation.

It was found that whereas the activity of the cathode decreased as the pressure was diminished, the anode showed a corresponding increase in activity.

* Communicated by the Physical Society : read March 1.3, 1908.

† Rutherford, Phil. Mag. Feb. 1900.

† Makower, Phil. Mag. Nov. 1905.

\$ Rutherford, Phil. Mag. Jan. 1903. 
Wood.

Phil.Mag. Ser 6. Vol.15, PLXVIII.
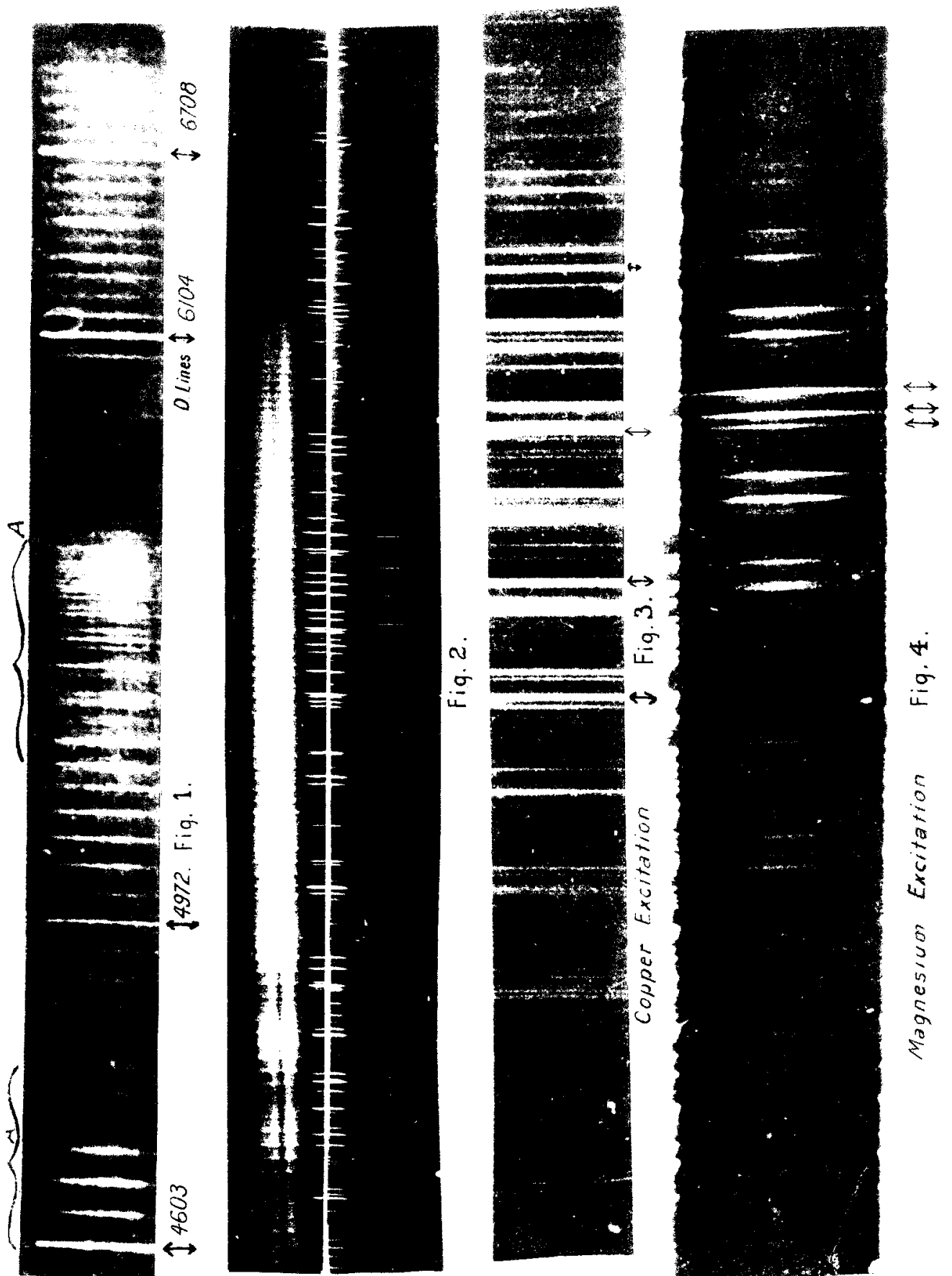

$N$

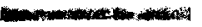

$\longleftrightarrow$

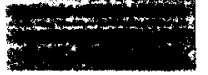

$$
\text { . }
$$

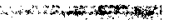
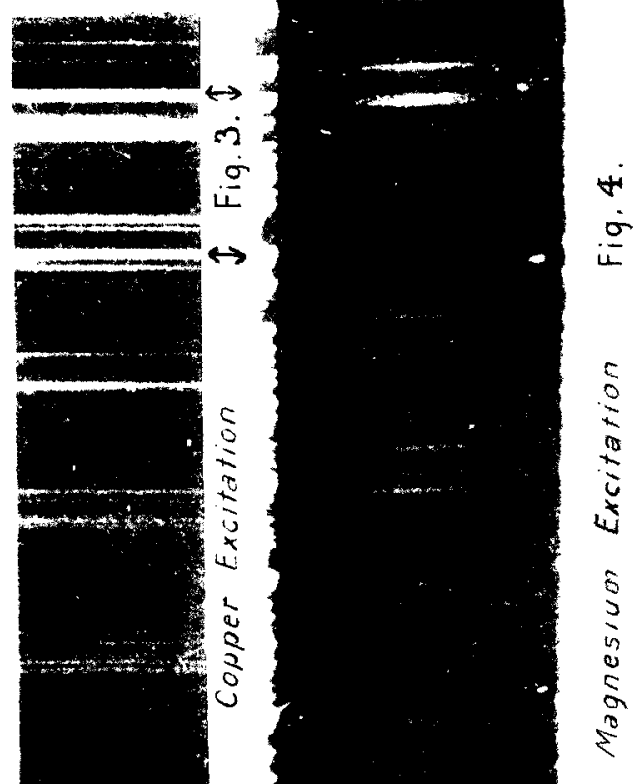


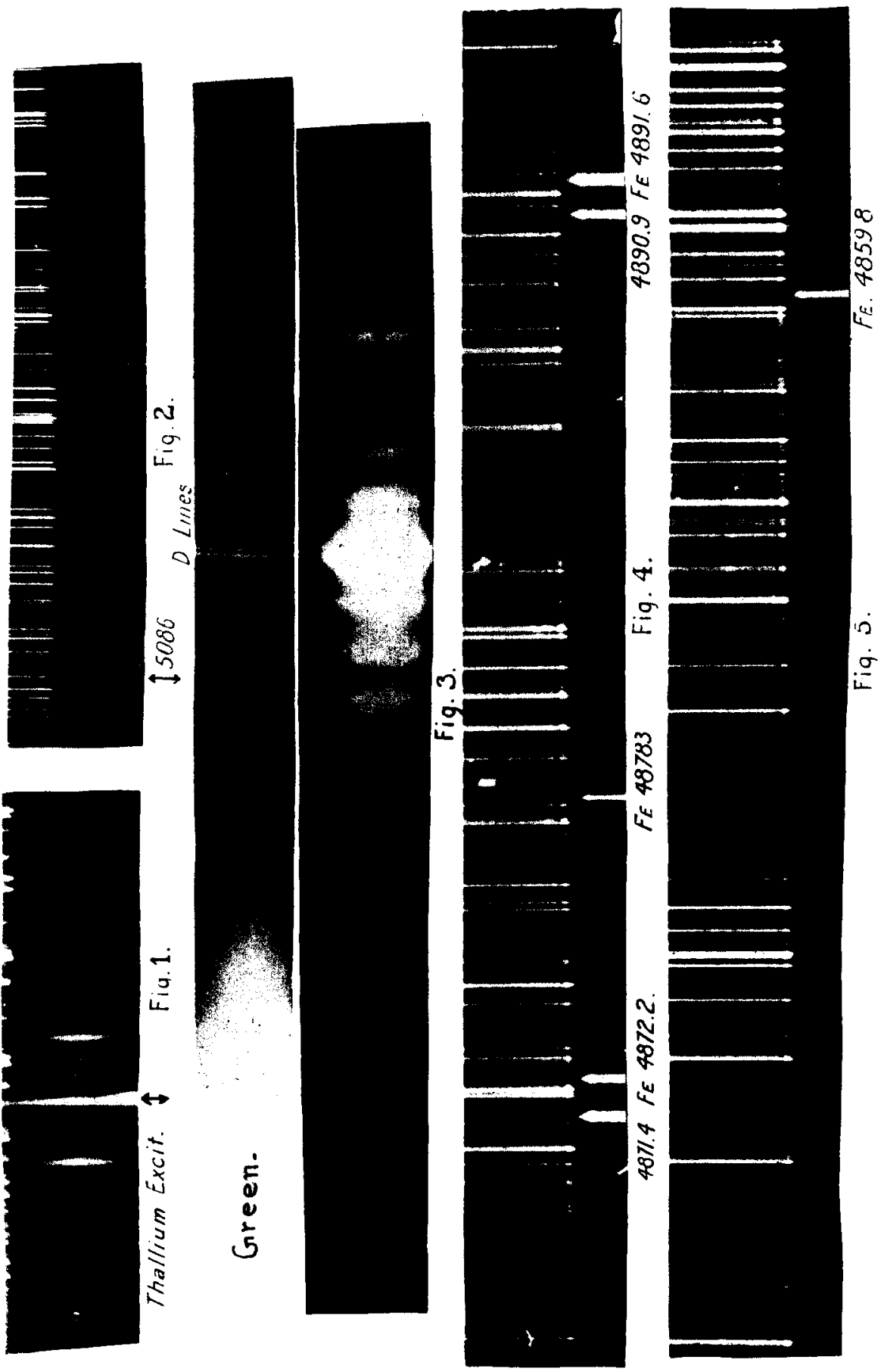

